\title{
Selection of Culture Medium and Incubation Time for Growth and Production of Beauvericin by Local Beauveria bassiana
}

\author{
Y. P. Roswanjaya ${ }^{1 *}$, N. A. Saryanah ${ }^{1}$, W. Nawfetrias ${ }^{1}$, H. Rosdayanti ${ }^{1}$ and A. L. Putri ${ }^{2}$ \\ ${ }^{1}$ Centre of technology for Agricultural Production, Agency for the Assessment and Application of Technology (BPPT), \\ Serpong, South Tangerang 15314, Indonesia \\ ${ }^{2}$ Research Centre for Biology, Indonesian Institute of Sciences (LIPI), Cibinong, Bogor 16911, Indonesia \\ ${ }^{*}$ Corresponding author. Email: yuda.purwana@bpt.go.id
}

\begin{abstract}
Beauveria bassiana, an entomopathogenic fungus, is a high producer of beauvericin (BEA). BEA is a proven and useful compound as a mycoinsecticide for plant pest control and a potential antifungal and anticancer agent for human. BEA produced by Beauveria bassiana fungi, mainly found as an intracellular product, means its production is dependent on the growth of the fungi in the culture medium. This study investigated four culture mediums and two incubation times to enhance growth and BEA production by Beauveria bassiana isolated from the infected insects in Kediri and Mojokerto, East Java, Indonesia. The four culture mediums were Potato Dextrose Broth (PDB), Yeast and Malt Extract Broth (YMB), Malt Extract Broth (MB), and Fusarium Defined Medium (FDM), and two incubation times were 6 and 12 days. The biomass and BEA production were studied in a batch culture without agitation. Our data shows that YMB was the optimum culture medium to produce high biomass of the fungal mycelium in both strains for 6 and 12 days of incubation. However, instead of in YMB, the highest BEA production for both strains was obtained from Beauveria bassiana grown in PDB for 6 days and in MB for 12 days. Correlation between biomass and BEA production in every culture medium is then calculated to see the BEA specific production. The highest BEA specific production has resulted from Beauveria bassiana grown for 12 days in MB medium with BEA yield was 103,42 mg/L and 237,49 mg/L for strain Kediri and Mojokerto, respectively.
\end{abstract}

Keywords: Beauveria bassiana, beauvericin, culture medium, incubation time, beauvericin specific production

\section{INTRODUCTION}

The hypocrealean fungus Beauveria bassiana (Bals.) Vuill. (Clavicipitaceae) is a well-known entomopathogen because of its ability to infect hundreds of host species belonging to most insect orders [1]. Distribution of this fungi is worldwide, found on infected insects both in temperate and tropical areas throughout the world. Besides its entomopathogenic ability, this fungi has also been shown to live saprophytically in the soil, to colonize the rhizosphere, to have antagonistic activities against plant pathogens [2], [3], as well as to grow endophytically inside plant tissues [4]. Based on the description by Rehner \& Buckley [5], B. bassiana is characterized by white, later yellowish, or occasionally reddish colonies. The reverse is uncolored, or yellowish to pinkish. Conidia cells consist of globose to flask-shaped basal part and up to $20-\mu \mathrm{m}$ long rachis, mostly forming a zig-zag. Conidia are hyaline, globose to broadly ellipsoidal, generally $2-3 \times 2-2.5 \mu \mathrm{m}$. The conidia are formed in clusters, like snowballs or cotton balls.

B. bassiana attack their host insects percutaneously, and the infection pathway of this fungi consist of several crucial steps [6]. The following steps are the attachment of the conidia to the cuticle, germination, penetration through the cuticle, overcoming the host response and immune defense reactions, proliferation within the host by the formation of hyphal bodies/blastospores, and saprophytic outgrowth from the dead host and production of new conidia. Invasion of the fungi is synergic with the host response activities, and these interactions are complex processes and comprise many molecular and cellular reactions [7]. The successful infection is marked by hyphal fungi that invade tissues of the host insect by extensive vegetative growth and the production of toxins. This process is followed by the death of the insect and the end of pathogenesis. 
B. bassiana produces several toxins in vitro and in vivo, but the most important toxin is beauvericin (BEA) [8], [9]. BEA is a cyclic hexadepsipeptide mycotoxin that belongs to the enniatin antibiotic family. It contains threeD-hydroxyisovaleryl and three N-methylphenylalanyl residues in alternating sequence [10], [11]. BEA has also already known produce by other fungi such as Isaria fumosorosea and Isaria farinose (formerly Paecilomyces fumosoroseus and Paecilomyces farinosus) [12] and by some Fusarium species [13]-[15]. The difference of BEA produced by $B$. bassiana with another fungi species is in the nature of the $\mathrm{N}$-methylamino acid, causing the difference in their bioactivities. Besides has insecticidal activity, BEA reported has antibacterial activity [16], [17], antifungal activity [18], [19], antiviral activity [20], and anticancer activity [21]-[23]. The broad range function of BEA has motivated several research studies on its application as a potential antibiotic and anticancer agent for human health care.

As a fungal metabolite, mycelial fermentation may be a feasible and efficient method to scale-up the production of BEA. The essential factor in developing an efficient fermentation process is the choice of culture medium for fungal growth. Nutrients and physicochemical conditions will be a major key to the successful high production of BEA. However, the report studies on the selection of culture medium for BEA production by $B$. bassiana is very limited. Instead of for B. bassiana, most of the studies were focused on investigating culture medium for Fusarium species [24][26]. As shown in those studies, the highest mycelia growth and BEA production were reached in Fusarium Defined Medium with a level of BEA was $0.2 \mathrm{~g} / \mathrm{L}$ [24]. Furthermore, increasing $9 \mathrm{~g}$ of glucose and $3 \mathrm{~g}$ of peptone from the Fusarium Basal Media was also increasing BEA level from $156 \mathrm{mg} / \mathrm{L}$ to $198 \mathrm{mg} / \mathrm{L}$ [26]. In addition, glucose feeding combined with resin to Fusarium Basal Media in the early stationary growth phase (day 7) was increasing BEA yield from 194 to $265 \mathrm{mg} / \mathrm{L}$ [25].

During B. bassiana or other fungi fermentation, BEA can be found mainly as an intracellular product. This fact suggests that BEA production is dependent on the fungal growth in the culture medium. However, at a high level, the accumulation of BEA within the fungal mycelia can cause growth inhibition due to its antibiotic properties. Because of this feedback inhibition, incubation time also plays an important role during the fermentation process. The maximal BEA concentration produced by Beauveria sp. FKI-1366 was achieved after 6 days of fermentation in the liquid culture medium [19]. Moreover, in Fusarium, Madry et al. [27] reported that the optimal harvesting time of BEA from mycelial culture was four days of fermentation. After this period, the production of biologically active compounds did not further increase.

Our laboratory has two local isolates of $B$. bassiana, isolated from the infected insects in cocoa (Theobroma cacao) plantation in Kediri and Mojokerto, East Java, Indonesia. In this present study, we investigated four different culture mediums for the maximal production of BEA in two incubation times for both isolates. Furthermore, BEA specific production was calculated to see the correlation between biomass and BEA production in every culture medium.

\section{MATERIAL AND METHODS}

\subsection{Microorganism and culture conditions}

B. bassiana was isolated from the infected insects in the cocoa plantation in Kediri and Mojokerto, East Java, Indonesia. Both fungi were identified as $B$. bassiana according to the morphological criteria described by Lacey [28]. Stock and working culture of B. bassiana strain Kediri and Mojokerto were maintained on solid Potato Dextrose Agar (PDA) at $28^{\circ} \mathrm{C}$. Fungal biomass and BEA production were studied in a batch culture without agitation at $28^{\circ} \mathrm{C}$. The inoculum was prepared by taking a full colony of $B$. bassiana from 6 days old of working culture with a corkborer. Three agar plates with $1 \mathrm{~cm}$ in diameter fully grown by $B$. bassiana were put aseptically to each $250 \mathrm{~mL}$ Erlenmeyer flask containing $100 \mathrm{~mL}$ of different culture mediums.

\subsection{Selection of culture medium}

For the selection of fungal growth and BEA production, both $B$. bassiana were cultivated in the following four types of complete culture medium. The detailed composition of every medium (amounts were listed per liter) were: 1) Potato Dextrose Broth (PDB): infused potato, $200 \mathrm{~g}$; and dextrose, 20 g. 2) Malt Extract Broth (MB): malt extract base, $6 \mathrm{~g}$; yeast maltose, $1.8 \mathrm{~g}$; dextrose, $6 \mathrm{~g}$; and yeast extract, $1.2 \mathrm{~g}$. 3) Yeast and Malt Extract Broth (YMB): malt extract base, $3 \mathrm{~g}$; yeast extract, $3 \mathrm{~g}$; peptone, $5 \mathrm{~g}$; and dextrose, $10 \mathrm{~g}$. 4) Fusarium Defined Medium (FDM): sucrose, $25 \mathrm{~g}$; $\mathrm{NaNO}_{3} 4.25 \mathrm{~g}$; NaCl, $5 \mathrm{~g}$ : $\mathrm{MgSO}_{4}$ $7 \mathrm{H}_{2} \mathrm{O}, 2.5 \mathrm{~g} ; \mathrm{KH}_{2} \mathrm{PO}_{4}, 1.36 \mathrm{~g} ; \mathrm{FeSO}_{4}-7 \mathrm{H}_{2} \mathrm{O}, 0.01 \mathrm{~g}$; and $\mathrm{ZnSO}_{4}-7 \mathrm{H}_{2} \mathrm{O}, 0.0029 \mathrm{~g}$.

\subsection{Determination of fresh biomass, BEA content, and BEA specific production}

B. bassiana mycelial mass was separated from the surface of the liquid medium and put on the filter paper, and then air-dried at room temperature until all liquid medium residues evaporated and mycelial mass became relatively dry. Biomass in the results was to represent the fresh weight of fungal biomass.

BEA extraction procedure followed the method developed by Moretti et al. [29] with modifications. For each $B$. bassiana culture, a $20 \mathrm{~g}$ sample was extracted in a blender with $100 \mathrm{~mL}$ of $\mathrm{MeOH}: 1 \%$ aqueous $\mathrm{NaCl}(55: 45)$ for 3 minutes and filtered through filter paper (Whatman No.1) using a vacuum pump. The filtrate $(50 \mathrm{~mL})$ was transferred into a separatory funnel and defatted twice using $50 \mathrm{~mL}$ of $\mathrm{n}$-hexane. The upper $\mathrm{n}$-hexane layer was discarded, and the methanol layer was extracted with chloroform $(3 \times 30 \mathrm{~mL})$. The $\mathrm{CH}_{2} \mathrm{Cl}_{2}$ extracts were collected, evaporated to dryness, dissolved in $1 \mathrm{~mL}$ of methanol, and then analyzed for BEA content. 
BEA was quantified by High Performance Liquid Chromatography (HPLC), as previously described by Logrieco et al. [30] with modifications. HPLC was carried out using a Symmetri® C18 $5 \mu \mathrm{m}, 150$ x $4.6 \mathrm{~mm}$ column, and a PDA UV detector, set at $225 \mathrm{~nm}$ using acetonitrile and water $(85: 5 \mathrm{v} / \mathrm{v})$ as an eluent, with a flow rate of $1.3 \mathrm{~mL} / \mathrm{min}$ under a pressure $5.5 \mathrm{MPa}$. Quantification by HPLC procedures was done by comparison of the peak height of BEA against a calibration curve of the peak height obtained with the BEA standard. BEA reference standard was purchased from Sigma Chemical Co.

BEA specific production was calculated by divided BEA content produced by fungi with the initial grams of the sample used for extraction, then multiple it with fresh biomass produced in the same medium and at the same time point. This normalization was conducted to see the real correlation between biomass and BEA production in every culture medium.

\subsection{Experiment design and statistical analysis}

This experiment was constructed by factorial completely randomized design on all four culture mediums and two incubation times to examine their effects on fungal growth for each isolate. The measurement of fresh biomass in this study was carried out in triplicate. However, the effects of different growth medium and incubation time on BEA content were carried out once without replication. We used $\mathrm{R}$ version 3.6.0 to conduct statistical tests. To compare fungal biomass among treatments, we tested the average of fresh biomass for significance differences with ANOVA and a post-hoc Duncan tests for pairwise comparison with a 95\% confidence interval.

\section{RESULTS}

\subsection{Growth of B. bassiana in different culture mediums}

In this experiment, we found that $B$. bassiana strain Kediri and Mojokerto were well grown in all mediums tested (Figure 1). The pattern of growth in every medium from both strains was similar; fresh biomass was significantly increased from 6to 12 days of incubation. However, B. bassiana strain Kediri shows better growth compared to strain Mojokerto (Figure 1 A \& B). The highest fresh biomass of both strains was reached when fungi were grown in YMB for 6 or 12days. Moreover, In strain Kediri, the highest increasing level of fresh biomass from 6 to 12days was also shown in YMB. In contrast, for strain Mojokerto, although YMB was the optimum medium to induce growth, the highest increasing fresh biomass level was in the MB medium with a value of 2,29 grams (Figure 1B).

The FDM specifically was formulated for the growth of Fusarium species, but our result shows that FDM was could also induce growth of B. bassiana, especially for strain Kediri. In this medium, the fresh biomass of mycelial was the second highest after YMB in both time points
(Figure 1A). However, we could not see this phenomenon in strain Mojokerto. Furthermore, PDB, as a general medium for fungal growth, did not show a significant effect for $B$. bassiana. This medium was effected fungal growth as high as the MB medium after 12days, much lower compared to YMB for both strains.
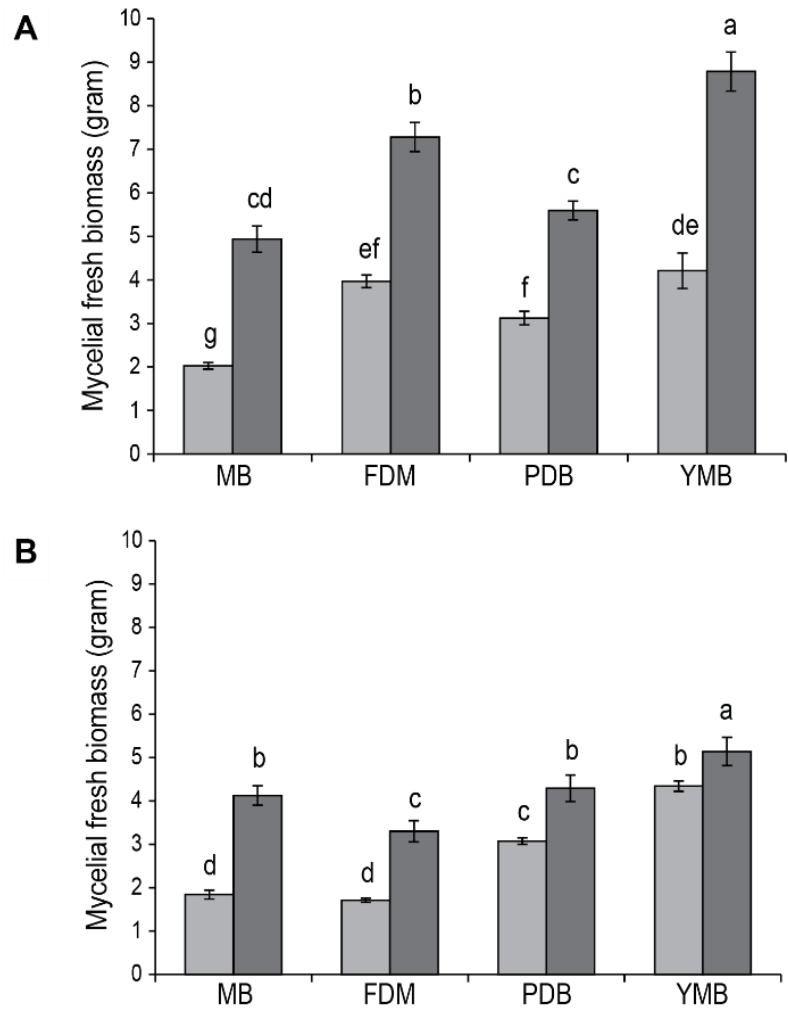

\section{$\square 6$ days $\square 12$ days}

Figure 1. Growth of B. bassiana strain Kediri (A) and Mojokerto (B) in four different culture mediums for 6 and 12 days of incubation. Different letters above the bars indicate statistical significance $(p<0.05)$ as determined by the ANOVA test in combination with Duncan's post-hoc test.

\subsection{Profile of BEA produced by B. bassiana in different culture mediums}

BEA can be isolated from both strains of $B$. basianna in all four culture mediums, even though the contents were highly variable (Figure 2 ). The highest BEA content has resulted from $B$. bassiana strain Kediri grown in PDB for 6days and in MB for 12days with the contents were 51 and $419 \mathrm{mg} / \mathrm{L}$, respectively (Figure 2A). A similar trend was also found in strain Mojokerto; the highest BEA content for 6dayshas resulted from $B$. bassiana grown in PDB (1402 mg/L), meanwhile for 12days was in MB (1151 $\mathrm{mg} / \mathrm{L}$ ) (Figure 2B). In general, the BEA content increased from 6 to 12days, except for the B. bassiana grown in PDB 
for both strains. In the PDB, there was a significant reduction in the BEA content from 6 to 12days, especially for strain Mojokerto. Our data also shows that in YMB, the BEA contents produced by both strains were relatively constant in the two-time points. To our surprise, although YMB was the optimum medium for B. bassiana growth, the effect of this medium on BEA content was weaker compared to other mediums. Furthermore, in our experiment, we also found that the production of BEA was low in FDM for both strains, which means that Fusarium specific medium can be used to grow B. bassiana, but this medium was failed to induce a high BEA content.
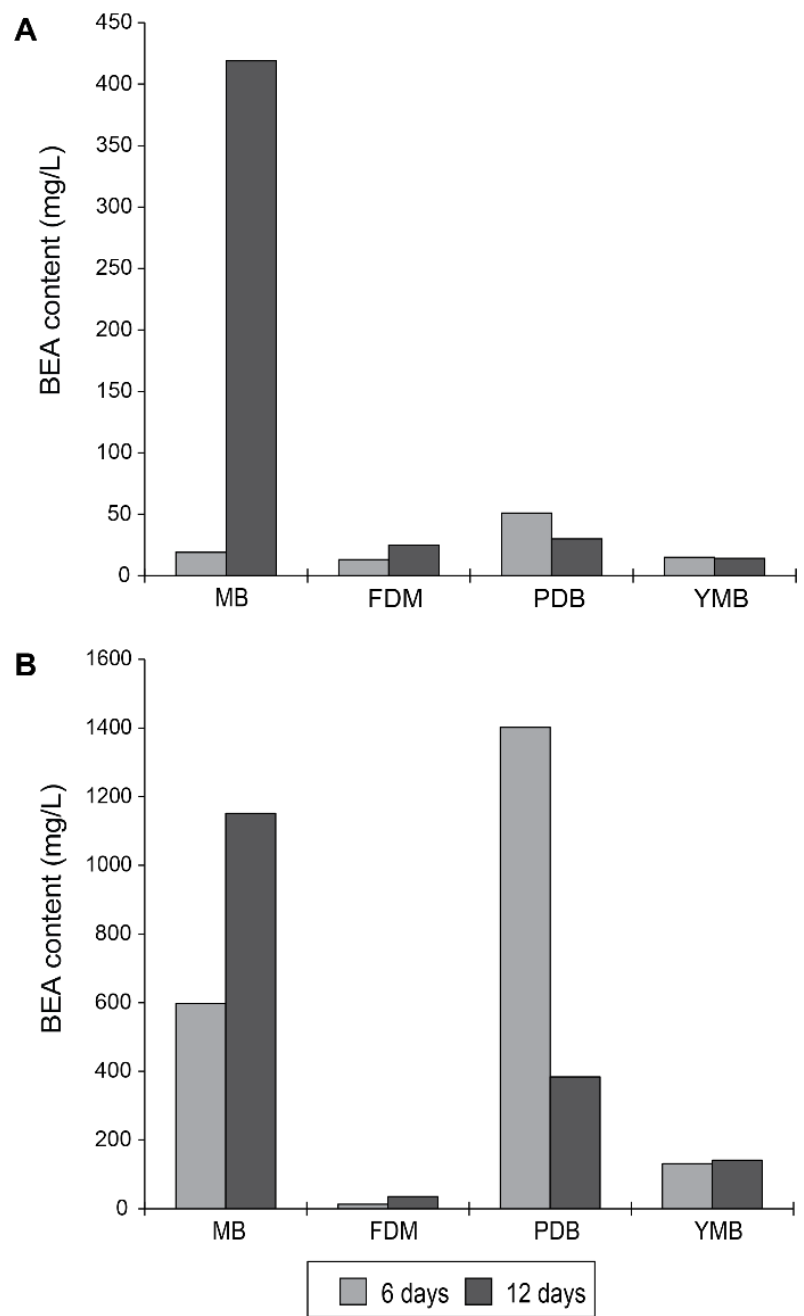

Figure 2. Profile of BEA content produced by $B$. bassiana strain Kediri (A) and Mojokerto (B) in four different culture mediums for 6 and 12 days of incubation.

\subsection{BEA specific production}

We calculated BEA specific production for our two strains in every culture medium and in both time points (Table 1). Fungal biomass was the crucial factor in this calculation as it can be seen that higher BEA specific production was affected by higher biomass. Our data shows this clear event in B.bassiana strain Kediri and Mojokerto grown in FDM for 6days. Although BEA content for both strains was similar $(13 \mathrm{mg} / \mathrm{L})$, but because their biomass was different, BEA specific production for strain Kediri was higher than strain Mojokerto (2,56 and 1,11 mg/L, respectively). Generally, the trends for BEA specific production in this experiment have followed the trends for BEA content, except for strain Kediri grown in PDB. There was a reduction of BEA content from day $6^{\text {th }}$ to $12^{\text {th }}$. Meanwhile, in their BEA specific production, the levels were relatively the same, 7,96 and $8,39 \mathrm{mg} / \mathrm{L}$ for 6 and 12 days of incubation.

Tablel 1. BEA specific production from B. bassiana strain Kediri and Mojokerto

\begin{tabular}{llcccccc}
\hline Strain & Medium & \multicolumn{2}{c}{$\begin{array}{c}\text { fresh biomass } \\
(\mathrm{g})\end{array}$} & \multicolumn{2}{c}{$\begin{array}{c}\text { BEA content } \\
(\mathrm{mg} / \mathrm{L})\end{array}$} & \multicolumn{2}{c}{$\begin{array}{c}\text { BEA Specific production } \\
(\mathrm{mg} / \mathrm{L})\end{array}$} \\
& & 6 days & 12 days & 6 days & 12 days & 6 days & 12 days \\
\hline Kediri & MB & 2,03 & 4,94 & 19 & 419 & 1,93 & 103,42 \\
& FDM & 3,97 & 7,28 & 13 & 25 & 2,58 & 9,10 \\
& PDB & 3,12 & 5,59 & 51 & 30 & 7,96 & 8,39 \\
& YMB & 4,21 & 8,78 & 15 & 14 & 3,16 & 6,15 \\
Mojokerto & MB & 1,84 & 4,13 & 598 & 1151 & 55,02 & 237,49 \\
& FDM & 1,71 & 3,30 & 13 & 35 & 1,11 & 5,78 \\
& PDB & 3,07 & 4,29 & 1402 & 384 & 215,44 & 82,37 \\
& YMB & 4,34 & 5,14 & 131 & 141 & 28,43 & 36,24 \\
\hline
\end{tabular}

\section{DISCUSSION}

In vitro BEA production by fungi $B$. bassiana is affected by many environmental factors such as culture medium, temperature, $\mathrm{pH}$ condition, and incubation time. The precise association among those factors will create an optimum condition for fungal growth and production of secondary metabolites, including BEA. To determine the best environmental factor for growth and BEA production of $B$. bassiana, we aimed to select a culture medium and incubation time trough selection of four different culture mediums and two incubation times. We did not investigate the $\mathrm{pH}$ condition and temperature in this experiment. As a consequence, we referred to some reports explaining this condition. Fargues et al., and Hallsworth \& Magan, have reported that the optimum temperature for $B$. bassiana growth was $28^{\circ} \mathrm{C}$ [31], [32], whereas the optimum $\mathrm{pH}$ medium was 7 [33]. In this paper, we showed that with a specific culture medium and with longer incubation time, fungal biomass and BEA content produced at a high level for both strains (Figure $1 \& 2$ ). 
Components of the nutrient in the medium used for growing $B$. bassiana or other fungi as general influence biomass yield and production of metabolites. Moreover, the most important component in a culture medium for this purpose is carbon and nitrogen. As an imperfect fungi Deuteromycetes, $B$. bassiana can be grown on a medium containing only simple carbon like sucrose, nitrogen, and mineral components [34]. Our result indicates that four culture mediums with different carbon and nitrogen sources can still be used to grow B. bassiana. The YMB, which contains double carbon (dextrose and malt extract) and nitrogen (yeast extract and peptone) sources, was the most optimum medium to produce high biomass in both strains. However, the biomass from strain Kediri is higher than strain Mojokerto. It suggests that the demand for a particular nutrient frequently varies not only within a single species but also for individual strains of a species. The increasing pattern of fungal biomass shows the similarity between our two strains in all mediums. Nevertheless, the differences in the biomass yield confirm that both strains were completely different.

In contrast to fungal biomass, YMB found as the weakest medium to induce BEA content in both strains, indicates that fungal growth was not linearly correlated with the BEA content. The use of different types of carbon and nitrogen sources in the four culture mediums are not clear to determine their effects on fungal biomass or BEA content. Our data only reveals that a medium can be the most optimum, but which components have a high impact are hard to specify. The way to know the effect of every component is by doing optimization on basal medium with various defined carbon and nitrogen sources. Optimization of the carbon/nitrogen ratio can be done by response surface methodology that has been proven to select the medium for growth and BEA production from Fusarium oxysporum KFCC 11363P [24]. However, in this experiment, we used only complete mediums to simplify the selection process. Even though without $\mathrm{C} / \mathrm{N}$ ratio optimization, our result shows that some medium was suitable for producing BEA with a high content in our two strains.

Observation of incubation time for fungal biomass yield indicates that prolonged incubation time will increase the biomass. This is a common phenomenon for microorganism growth. As long as still in the exponential phase, microorganisms will continue to grow until a particular time [35]. In this experiment, the fresh biomass of both strains was increased from 6 to 12 days of incubation, confirm that from 6 to 12 days are still in the range of the exponential phase. In the case of BEA content, our data shows that between time points observed, 12 days was the best incubation time compared to 6 days. This result was opposite to Fukuda et al. [19], which reported that maximal BEA content was reached after 6 days of fermentation. They did not observe BEA content from Beauveria sp. FK1-1366 after 6 days, so we do not know the pattern of BEA content produced by this Beauveria species after 6 days. In contrast, BEA contents in our observation were also high on day $6^{\text {th }}$, but after 12 days, the levels were even higher in almost all culture mediums. This result was also contradictive with Madry [27] that foundafter four days of fermentation, BEA content did not further increase.

Among the mediums we used, PDB showed an induction to BEA content that differs from another medium. The BEA content was higher on day $6^{\text {th }}$, then decrease significantly on day $12^{\text {th }}$, whereas the other mediums show the opposite results (Figure 2A \& B). The BEA content reductionin this medium might be caused by the initial BEA content that wasalready high on day $6^{\text {th }}$, indicates that after reach a maximal level, BEA content decreases over time. This finding confirms the results from $\mathrm{Xu}$ et al. [25] that showed BEA at a high level has negative feedback to the downstream of another BEA production cycle in a longer incubation.

Since the trend of BEA content is similar to BEA specific production, which was affected by biomass, we found that although grown in the same medium, the ability of the two strains used in this experiment is different in producing BEA. Our BEA contents and BEA specific production comparison between two strains revealed that strain Mojokerto was stronger in producing BEA in all four mediums and in both time points than strain Kediri. This significant difference might be affected by strain specificity, support the hypothesis that BEA content was strain-dependent [36]. Even though in the same species, a different strain can produce BEA content differently.

\section{CONCLUSION}

The medium needed for optimum growth and BEA production is different. These differences could help us to select which medium to be used based on our goals. YMB is the optimum medium to yield high biomass, whereas MB is the most suitable medium to produce high BEA content. Additionally, 12 days of incubation is the optimum incubation time for both purposes. The selection results obtained from this study may be useful for the efficient and economical production of BEA on batch culture fermentation.

\section{AUTHORS' CONTRIBUTIONS}

YPR and NAS planned and designed the research; YPR, NAS, WN, HR, and ALP performed the experiment and analyzed the data; and YPR wrote the manuscript.

\section{ACKNOWLEDGMENT}

The authors like to thank the staff at Nusantara Plantation Company (PTPN) XII Kediri andthe Plantation office of Mojokerto Regency for help with sample collection. The authors are grateful to Lira Hikaru for guiding in HPLC analysis. This work was supported by the Issuance of spending authority (DIPA) BPPT to the Centre of Technology of Agricultural Production under the "Optimization of Cocoa Cultivation TechniquesTrough Pest Control Management” Project. 


\section{REFERENCES}

[1] F. E. Vega et al., 'Fungal entomopathogens: new insights on their ecology', Fungal Ecology, vol. 2, no. 4, pp. 149-159, Nov. 2009, doi: 10.1016/j.funeco.2009.05.001.

[2] E. Quesada-Moraga, C. López-Díaz, and B. B. Landa, 'The Hidden Habit of the Entomopathogenic Fungus Beauveria bassiana: First Demonstration of Vertical Plant Transmission', PLOS ONE, vol. 9, no. 2, p. e89278, Feb. 2014, doi: 10.1371/journal.pone.0089278.

[3] I. Garrido-Jurado, M. Fernández-Bravo, C. Campos, and E. Quesada-Moraga, 'Diversity of entomopathogenic Hypocreales in soil and phylloplanes of five Mediterranean cropping systems', Journal of Invertebrate Pathology, vol. 130, pp. 97-106, Sep. 2015, doi: 10.1016/j.jip.2015.06.001.

[4] F. E. Vega, F. Posada, M. Catherine Aime, M. PavaRipoll, F. Infante, and S. A. Rehner, 'Entomopathogenic fungal endophytes', Biological Control, vol. 46, no. 1, pp. 72-82, Jul. 2008, doi: 10.1016/j.biocontrol.2008.01.008.

[5] S. A. Rehner and E. Buckley, 'A Beauveria phylogeny inferred from nuclear ITS and EF1- $\alpha$ sequences: evidence for cryptic diversification and links to Cordyceps teleomorphs', Mycologia, vol. 97, no. 1 , pp. 84-98, Mar. 2005, doi: 10.1080/15572536.2006.11832842.

[6] G. Zimmermann, 'Review on safety of the entomopathogenic fungi Beauveria bassiana and Beauveria brongniartii', Biocontrol Science and Technology, vol. 17, no. 6, pp. 553-596, Jun. 2007, doi: 10.1080/09583150701309006.

[7] A. Vilcinskas and P. Götz, 'Parasitic Fungi and their Interactions with the Insect Immune System', in Advances in Parasitology, vol. 43, J. R. Baker, R. Muller, and D. Rollinson, Eds. Academic Press, 1999, pp. 267-313.

[8] H. Strasser, A. Vey, and T. M. Butt, 'Are There any Risks in Using Entomopathogenic Fungi for Pest Control, with Particular Reference to the Bioactive Metabolites of Metarhizium, Tolypocladium and Beauveria species?', Biocontrol Science and Technology, vol. 10, no. 6, pp. 717-735, Dec. 2000, doi: 10.1080/09583150020011690.

[9] T. M. Butt, C. Jackson, and N. Magan, Fungi As Biocontrol Agents: Progress Problems and Potential. CABI, 2001.

[10] R. L. Hamill, C. E. Higgens, H. E. Boaz, and M. Gorman, 'The structure op beauvericin, a new depsipeptide antibiotic toxic to artemia salina', Tetrahedron Letters, vol. 10, no. 49, pp. 4255-4258, Jan. 1969, doi: 10.1016/S0040-4039(01)88668-8.

[11] A. Logrieco et al., 'Beauvericin Production by FusariumSpecies', Appl. Environ. Microbiol., vol. 64, no. 8, pp. 3084-3088, Aug. 1998, doi: 10.1128/AEM.64.8.3084-3088.1998.
[12] Q. Weng, X. Zhang, W. Chen, and Q. Hu, 'Secondary Metabolites and the Risks of Isaria fumosorosea and Isaria farinosa', Molecules, vol. 24, no. 4, Art. no. 4, Jan. 2019, doi: 10.3390/molecules24040664.

[13] S. Gupta, S. B. Krasnoff, N. L. Underwood, J. A. A. Renwick, and D. W. Roberts, 'Isolation of beauvericin as an insect toxin from Fusarium semitectum and Fusarium moniliforme var. subglutinans', Mycopathologia, vol. 115, no. 3, pp. 185-189, Sep. 1991, doi: 10.1007/BF00462223.

[14] A. Logrieco, A. Rizzo, R. Ferracane, and A. Ritieni, 'Occurrence of Beauvericin and Enniatins in Wheat Affected by Fusarium avenaceum Head Blight', Appl. Environ. Microbiol., vol. 68, no. 1, pp. 82-85, Jan. 2002, doi: 10.1128/AEM.68.1.82-85.2002.

[15] J. Fotso, J. F. Leslie, and J. S. Smith, 'Production of Beauvericin, Moniliformin, Fusaproliferin, and Fumonisins B1, B2, and B3 by Fifteen Ex-Type Strains of Fusarium Species', Appl. Environ. Microbiol., vol. 68, no. 10, pp. 5195-5197, Oct 2002, doi: 10.1128/AEM.68.10.5195-5197.2002.

[16] L. A. Castlebury, J. B. Sutherland, L. A. Tanner, A. L. Henderson, and C. E. Cerniglia, 'Use of a bioassay to evaluate the toxicity of beauvericin to bacteria', World Journal of Microbiology and Biotechnology, vol. 15, no. 1, pp. 119-121, Feb. 1999, doi: 10.1023/A:1008895421989.

[17] G. Meca, I. Sospedra, J. M. Soriano, A. Ritieni, A. Moretti, and J. Mañes, 'Antibacterial effect of the bioactive compound beauvericin produced by Fusarium proliferatum on solid medium of wheat', Toxicon, vol. 56, no. 3, pp. 349-354, Sep. 2010, doi: 10.1016/j.toxicon.2010.03.022.

[18] L. Zhang et al., 'High-throughput synergy screening identifies microbial metabolites as combination agents for the treatment of fungal infections', PNAS, vol. 104, no. 11, pp. 4606-4611, Mar. 2007, doi: 10.1073/pnas.0609370104.

[19] T. Fukuda, M. Arai, Y. Yamaguchi, R. Masuma, H. Tomoda, and S. Omura, 'New Beauvericins, Potentiators of Antifungal Miconazole Activity, Produced by Beauveria sp. FKI-1366 I. Taxonomy, Fermentation, Isolation and Biological Properties', $J$. Antibiot., vol. 57, no. 2, pp. 110-116, Feb. 2004, doi: 10.7164/antibiotics.57.110.

[20] C.-G. Shin, D.-G. An, H.-H. Song, and C. Lee, 'Beauvericin and enniatins H, I and MK1688 are new potent inhibitors of human immunodeficiency virus type-1 integrase', The Journal of Antibiotics, vol. 62, no. 12, Art. no. 12, Dec. 2009, doi: 10.1038/ja.2009.102.

[21] G.-M. Jow, C.-J. Chou, B.-F. Chen, and J.-H. Tsai, 'Beauvericin induces cytotoxic effects in human acute lymphoblastic leukemia cells through cytochrome c release, caspase 3 activation: the causative role of calcium', Cancer Letters, vol. 216 , no. 2, pp. 165-173, Dec. 2004, doi: 10.1016/j.canlet.2004.06.005. 
[22] H.-I. Lin et al., 'Involvement of Bcl-2 family, cytochrome $\mathrm{c}$ and caspase 3 in induction of apoptosis by beauvericin in human non-small cell lung cancer cells', Cancer Letters, vol. 230, no. 2, pp. 248-259, Dec. 2005, doi: 10.1016/j.canlet.2004.12.044.

[23] L. Calò et al., 'Cytotoxic effects of the mycotoxin beauvericin to human cell lines of myeloid origin', Pharmacological Research, vol. 49, no. 1, pp. 73-77, Jan. 2004, doi: 10.1016/j.phrs.2003.07.002.

[24] H.-S. Lee, H.-H. Song, J.-H. Ahn, C.-G. Shin, G.-P. Lee, and C. Lee, 'Statistical Optimization of Growth Medium for the Production of the Entomopathogenic and Phytotoxic Cyclic Depsipeptide Beauvericin from Fusarium oxysporum KFCC 11363P', Journal of Microbiology and Biotechnology, vol. 18, no. 1, pp. 138-144, 2008.

[25] L.-J. Xu, Y.-S. Liu, L.-G. Zhou, and J.-Y. Wu, 'Enhanced beauvericin production with in situ adsorption in mycelial liquid culture of Fusarium redolens Dzf2', Process Biochemistry, vol. 44, no. 10, pp. 1063-1067, Oct. 2009, doi: 10.1016/j.procbio.2009.05.004

[26] L.-J. Xu, Y.-S. Liu, L.-G. Zhou, and J.-Y. Wu, 'Optimization of a liquid medium for beauvericin production in Fusarium redolens Dzf2 mycelial culture', Biotechnol Bioproc E, vol. 15, no. 3, pp. 460-466, Jun. 2010, doi: 10.1007/s12257-009-30312.

[27] N. Madry, R. Zocher, and H. Kleinkauf, 'Enniatin production by Fusarium oxysporum in chemically defined media', European J. Appl. Microbiol. Biotechnol., vol. 17, no. 2, pp. 75-79, Mar. 1983, doi: 10.1007/BF00499854.

[28] L. A. Lacey, Manual of Techniques in Insect Pathology. Academic Press, 1997.

[29] A. Moretti, A. Logrieco, A. Bottalico, A. Ritieni, G. Randazzo, and P. Corda, 'Beauvericin production by Fusarium subglutinans from different geographical areas', Mycological Research, vol. 99, no. 3, pp.
282-286, Mar. 1995, doi: 10.1016/S09537562(09)80899-X.

[30] Antonio. Logrieco et al., 'Natural occurrence of beauvericin in preharvest Fusarium subglutinans infected corn ears in Poland', J. Agric. Food Chem., vol. 41, no. 11, pp. 2149-2152, Nov. 1993, doi: 10.1021/jf00035a061.

[31] J. Fargues, M. S. Goettel, N. Smits, A. Ouedraogo, and M. Rougier, 'Effect of temperature on vegetative growth of Beauveria bassiana isolates from different origins', Mycologia, vol. 89, no. 3, pp. 383-392, May 1997, doi: 10.1080/00275514.1997.12026797.

[32] J. E. Hallsworth and N. Magan, 'Water and Temperature Relations of Growth of the Entomogenous Fungi Beauveria bassiana, Metarhizium anisopliae, and Paecilomyces farinosus', Journal of Invertebrate Pathology, vol. 74, no. 3, pp. 261-266, Nov. 1999, doi: 10.1006/jipa.1999.4883.

[33] A. Karthikeyan, V. Shanthi, and A. Nagasathya, 'EFFECT OF DIFFERENT MEDIA AND pH ON THE GROWTH OF BEAUVERIA BAS', p. 3, 2008.

[34] U. S. Iskandarov, A. G. Guzalova, and K. D. Davranov, 'Effects of nutrient medium composition and temperature on the germination of conidia and the entomopathogenic activity of the fungi Beauveria bassiana and Metarhizium anisopliae', Appl Biochem Microbiol, vol. 42, no. 1, pp. 72-76, Jan. 2006, doi: 10.1134/S000368380601011X.

[35] I. Málek and Z. Fenel, Theoretical and Methodological Basis of Continuous Culture of Microorganisms. Elsevier, 2013.

[36] Q. Wang and L. Xu, 'Beauvericin, a Bioactive Compound Produced by Fungi: A Short Review', Molecules, vol. 17, no. 3, Art. no. 3, Mar. 2012, doi: 10.3390/molecules 17032367 . 